\title{
Microscopic Study of the Development of the Optic Cup of the Green Turtle, Chelonia mydas in Oman
}

\author{
T. A. Ba-Omar; I.Y. Mahmoud; T. Al-Hinai and S. N. Al-Bahry \\ Department of Biology, College of Science, Sultan Qaboos University, P.O.Box 36 Muscat 123, \\ OMAN.
}

The green turtle population at Ras Al-Hadd, Reserve - Oman ranked second in the world and nest year round. Early morphogenesis of the eye in the turtle usually appears during early stages of the embryogenesis.

Eggs were randomly collected from 13 different nests from Ras Al-Hadd Reserve - Oman during June and transferred in plastic pockets with sand to the laboratory within 16 hours of oviposition. Each egg was incubated in a $600 \mathrm{ml}$ plastic container with a plastic cover $10 \mathrm{~cm}$ in diameter according to Mrosovsky (1988) method, with a minor modification. The eggs were placed in Precision Sanyo incubators adjusted at $300 \mathrm{C}$.

Eggs were opened on day 6 of incubation and the embryos were fixed in Karnovesky for examination with scanning electron microscopy (SEM). The tissue were examined under JEOL JSM-5600LV SEM operated at $10 \mathrm{kV}$.

The eye cup was developed on day 6 and was completely formed by day 14 with presumptive retinal pigment and retina (Fig. 1 and 2). However no further differentiation in the mantle layer was observed at this stage, in the photoreceptors or bipolar layers.

Reference:

1. Mrosovsky, N. 1988. Pivotal temperatures for loggerhead turtles (Caretta caretta) from northern and southern nesting beaches. Candian Journal of Zoology. 66: 661-669. 


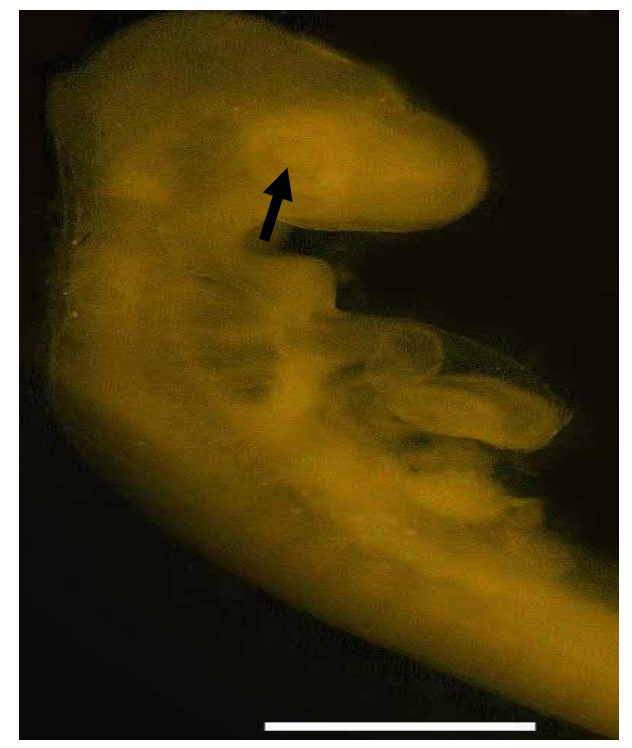

Fig 1. Day 7 of incubation of the turtle embryo showing the optic cup with visible lens ( arrow). Scale bar $=0.1 \mathrm{~cm}$.

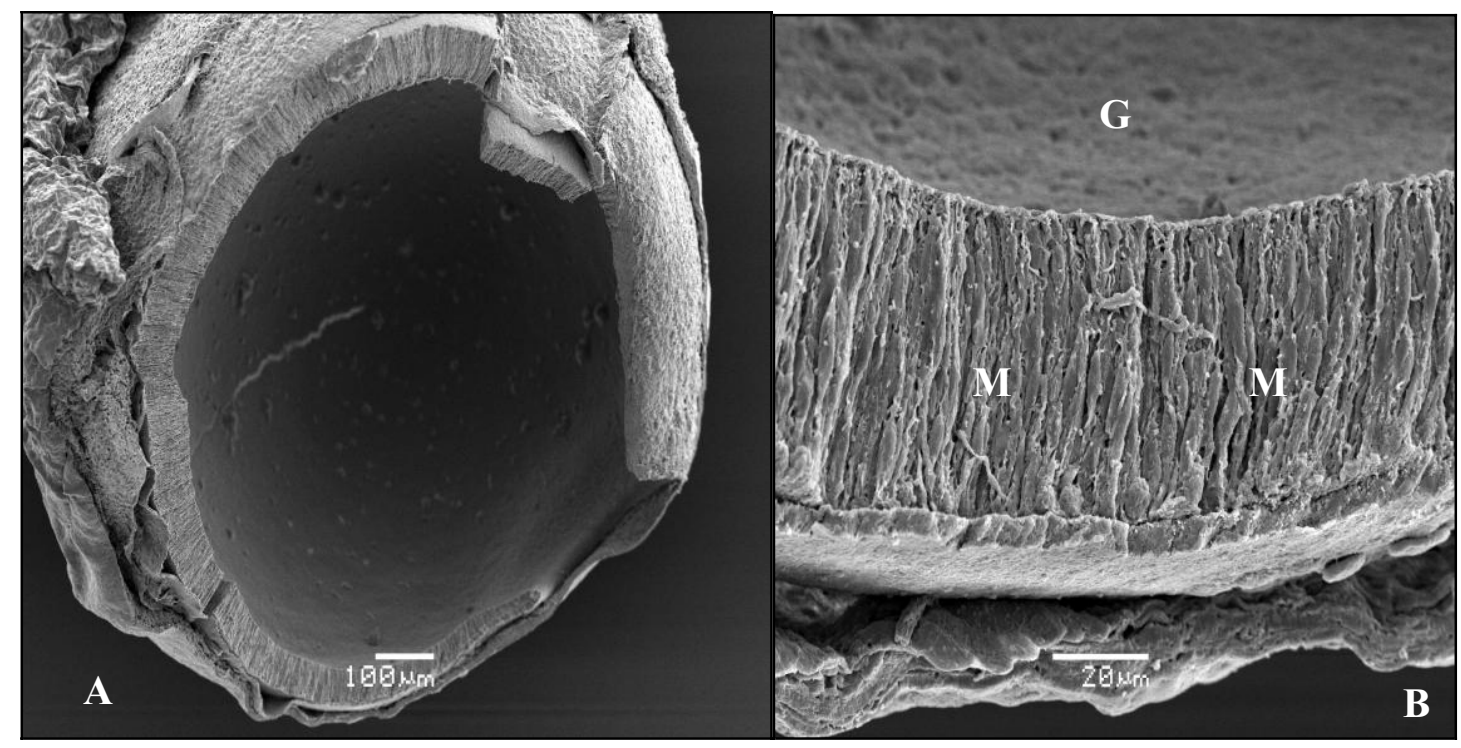

Fig. 2. Scanning electron micrographs of day 14 of incubation showing; (A) Fully formed eye cup; (B) Retina showing the beginning of differentiation of the ganglionic cells (G) and mantle layer $(\mathrm{M})$. 